\title{
Nice Point Sets Can Have Nasty Delaunay Triangulations*
}

\author{
Jeff Erickson \\ University of Illinois, Urbana-Champaign \\ jeffe@cs.uiuc.edu \\ http://www.cs.uiuc.edu/ jeffe
}

\begin{abstract}
We consider the complexity of Delaunay triangulations of sets of points in $\mathbb{R}^{3}$ under certain practical geometric constraints. The spread of a set of points is the ratio between the longest and shortest pairwise distances. We show that in the worst case, the Delaunay triangulation of $n$ points in $\mathbb{R}^{3}$ with spread $\Delta$ has complexity $\Omega\left(\min \left\{\Delta^{3}, n \Delta, n^{2}\right\}\right)$ and $\mathrm{O}\left(\min \left\{\Delta^{4}, \mathrm{n}^{2}\right\}\right)$. For the case $\Delta=\Theta(\sqrt{n})$, our lower bound construction consists of a uniform sample of a smooth convex surface with bounded curvature. We also construct a family of smooth connected surfaces such that the Delaunay triangulation of any good point sample has near-quadratic complexity.
\end{abstract}

\section{Introduction}

Delaunay triangulations and Voronoi diagrams are used as a fundamental tool in several geometric application areas, including finite-element mesh generation $[16,21$, 27, 28], deformable surface modeling [15], and surface reconstruction $[1,3,4,5,12,26]$. Many algorithms in these application domains begin by constructing the Delaunay triangulation of a set of $n$ points in $\mathbb{R}^{3}$. Delaunay triangulations can have complexity $\Omega\left(n^{2}\right)$ in the worst case, and as a result, all these algorithms have worst-case running time $\Omega\left(n^{2}\right)$. However, this behavior is almost never observed in practice except for highly-contrived inputs. For all practical purposes, three-dimensional Delaunay triangulations appear to have linear complexity.

One way to explain this frustrating discrepancy between theoretical and practical behavior would be to identify geometric constraints that are satisfied by real-world input and analyze Delaunay triangulations under those constraints. These constraints would be

*Portions of this work were done while the author was visiting INRIA, Sophia-Antipolis, with the support of a UIUC/CNRS/INRIA travel grant. This research was also partially supported by a Sloan Fellowship and by NSF CAREER grant CCR-0093348. See http://www.cs.uiuc.edu/ ${ }^{\sim}$ jeffe/pubs/ spread.html for the most recent version of this paper. similar to the realistic input models such as fatness or simple cover complexity, which many authors have used to develop geometric algorithms with good practical performance $[8,29]$. Unlike these works, however, our (immediate) goal is not to develop new algorithms, but rather to formally explain the good practical performance of existing code.

Dwyer $[20,19]$ showed that if a set of points is generated uniformly at random from the unit ball, its Delaunay triangulation has linear expected complexity. Golin and $\mathrm{Na}$ [24] recently derived a similar result for random points on the surface of a three-dimensional convex polytope. Although these results are encouraging, they are unsatisfying as an explanation of practical behavior. Real-world surface data generated by laser range finders, digital cameras, tomographic scanners, and similar input devices is often highly structured.

This paper considers the complexity of Delaunay triangulations under two types of practical geometric constraints. First, in Section 2, we consider the worstcase Delaunay complexity as a function of both the number of points and the spread - the ratio between its diameter and the distance between its closest pair. For any $n$ and $\Delta$, we construct a set of $n$ points with spread $\Delta$ whose Delaunay triangulation has complexity $\Omega\left(\min \left\{\Delta^{3}, n \Delta, n^{2}\right\}\right)$. When $\Delta=\Theta(\sqrt{n})$, our lower bound construction consists of a grid-like sample of a right circular cylinder with constant height and radius. We also show that the worst-case complexity of a Delaunay triangulation is $\mathrm{O}\left(\min \left\{\Delta^{4}, \mathrm{n}^{2}\right\}\right)$.

An important application of Delaunay triangulations that has received a lot of attention recently is surface reconstruction-given a set of points from a smooth surface $\Sigma$, reconstruct an approximation of $\Sigma$. Several algorithms provably reconstruct surfaces if the input points satisfy certain sampling conditions $[4,5,12,26]$. In Section 3, we consider the complexity of Delaunay triangulations of good samples of smooth surfaces. Not surprisingly, oversampling almost any surface can produce a point set whose Delaunay triangulation has 
quadratic complexity. We show that even surface data with no oversampling can have quadratic Delaunay triangulations and that there are smooth surfaces where every good sample has near-quadratic Delaunay complexity. We also derive similar results for randomly distributed points on non-convex smooth surfaces.

We will analyze the complexity of three-dimensional Delaunay triangulations by counting the number of edges. Two points are joined by an edge in the Delaunay triangulation of a set $S$ if and only if they lie on a sphere with no points of $S$ in its interior. Since every vertex figure is a planar graph, Euler's formula implies that a Delaunay triangulation with $n$ vertices and $e$ edges has at most $2 e-2 n$ triangles and $e-n$ tetrahedra.

In the interest of saving space, several straightforward but tedious calculations are omitted from this extended abstract.

\section{Sublinear Spread}

We define the spread $\Delta$ of a set of points (also called the distance ratio [17]) as the ratio between the longest and shortest pairwise distances. In this section, we derive upper and lower bounds on the worst-case complexity of the Delaunay triangulation of a point set in $\mathbb{R}^{3}$, as a function of both the number of points and the spread.

If the spread takes its minimum value $\Theta\left(n^{1 / 3}\right)$, the points are packed into a tight lattice, and the Delaunay triangulation has only linear complexity. On the other hand, all known examples of point sets with quadraticcomplexity Delaunay triangulations have spread $\Omega(n)$. Thus, it is natural to ask how the worst-case complexity of the Delaunay triangulation changes as the spread varies between these two extremes. The spread of a set of points is loosely related to its dimensionality. If a set uniformly covers a bounded region of space, a surface of bounded curvature, or a curve of bounded curvature, its spread is respectively $\Theta\left(n^{1 / 3}\right), \Theta\left(n^{1 / 2}\right)$, or $\Theta(n)$. The case of surface data is particularly interesting in light of numerous algorithms that reconstruct surfaces using a subcomplex of the Delaunay triangulation. We will discuss surface reconstruction in more detail in the next section.

\section{$2.1 \quad$ Lower Bounds}

The crucial special case of our lower bound construction is $\Delta=\Theta(\sqrt{n})$. For any positive integer $x$, let $[x]$ denote the set $\{1,2, \ldots, x\}$. Our construction consists of $n$ evenly spaced points on a helical space curve:

$$
S_{\sqrt{n}}=\left\{\left(\frac{t}{n}, \cos \frac{t}{\sqrt{n}}, \sin \frac{t}{\sqrt{n}}\right) \mid t \in[n]\right\} .
$$

See Figure 1. As we show below, the Delaunay triangulation of $S_{\sqrt{n}}$ has complexity $\Omega\left(n^{3 / 2}\right)$. Note that $S$ is a grid-like uniform $\varepsilon$-sample of a right circular cylinder, where $\varepsilon=\Theta(\sqrt{1 / n})$. By adding additional points on two hemispherical caps at the ends of the cylinder, we can extend $S$ into a uniform sample of a smooth convex surface with bounded curvature and constant local feature size.

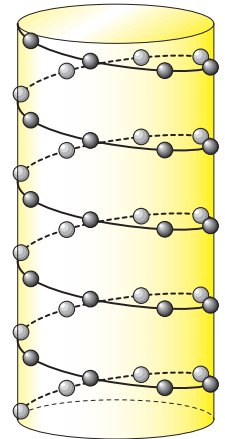

Figure 1. A set of $n$ points whose Delaunay triangulation has complexity $\Omega\left(n^{3 / 2}\right)$

Let $h_{\alpha}(t)$ denote the helix $(\alpha t, \cos t, \sin t)$, where $\alpha>0$ is a fixed parameter called the pitch. Using elementary trigonometric identities and matrix operations, we can simplify the insphere determinant for five points on this helix as follows.

$$
\begin{gathered}
\left|\begin{array}{cccccc}
1 & \alpha t_{1} & \cos t_{1} & \sin t_{1} & \alpha^{2} t_{1}^{2}+\cos ^{2} t_{1}+\sin ^{2} t_{1} \\
1 & \alpha t_{2} & \cos t_{2} & \sin t_{2} & \alpha^{2} t_{2}^{2}+\cos ^{2} t_{2}+\sin ^{2} t_{2} \\
1 & \alpha t_{3} & \cos t_{3} & \sin t_{3} & \alpha^{2} t_{3}^{2}+\cos ^{2} t_{3}+\sin ^{2} t_{3} \\
1 & \alpha t_{4} & \cos t_{4} & \sin t_{4} & \alpha^{2} t_{4}^{2}+\cos ^{2} t_{4}+\sin ^{2} t_{4} \\
1 & \alpha t_{5} & \cos t_{5} & \sin t_{5} & \alpha^{2} t_{5}^{2}+\cos ^{2} t_{5}+\sin ^{2} t_{5}
\end{array}\right| \\
=\alpha^{3}\left|\begin{array}{lllll}
1 & t_{1} & \cos t_{1} & \sin t_{1} & t_{1}^{2} \\
1 & t_{2} & \cos t_{2} & \sin t_{2} & t_{2}^{2} \\
1 & t_{3} & \cos t_{3} & \sin t_{3} & t_{3}^{2} \\
1 & t_{4} & \cos t_{4} & \sin t_{4} & t_{4}^{2} \\
1 & t_{5} & \cos t_{5} & \sin t_{5} & t_{5}^{2}
\end{array}\right|
\end{gathered}
$$

We obtain the surprising observation that changing the pitch $\alpha$ of the helix does not change the combinatorial structure of the Delaunay triangulation of any set of points on the helix. (More generally, scaling any set of points on any circular cylinder along the cylinder's axis leaves the Delaunay triangulation invariant.) Thus, for purposes of analysis, it suffices to consider the case $\alpha=1$. Let $h(t)=h_{1}(t)=(t, \cos t, \sin t)$.

Our first important observation is that any set of points on a single turn of any helix has a neighborly Delaunay triangulation, meaning that every pair of points is connected by a Delaunay edge. For any real value $t$, we define the bitangent sphere $\beta(t)$ as the 
unique sphere passing through $h(t)$ and $h(-t)$ and tangent to the helix at those two points.

Lemma 2.1. For any $0<t<\pi$, the sphere $\beta(t)$ intersects the helix $h$ only at its two points of tangency.

Proof: Symmetry considerations imply that the bitangent sphere must be centered on the $y$-axis, so it can be described by the equation $x^{2}+(y-a)^{2}+z^{2}=r^{2}$ for some constants $a$ and $r$. Let $\gamma$ denote the intersection curve of $\beta(t)$ and the cylinder $y^{2}+z^{2}=1$. Every intersection point between $\beta(t)$ and the helix must lie on $\gamma$. If we project the helix and the intersection curve to the $x y$ plane, we obtain the sinusoid $y=\cos x$ and a portion of the parabola $y=\gamma(x)=\left(x^{2}-r^{2}+a^{2}+1\right) / 2 a$. These two curves meet tangentially at the points $(t, \cos t)$ and $(-t, \cos t)$.

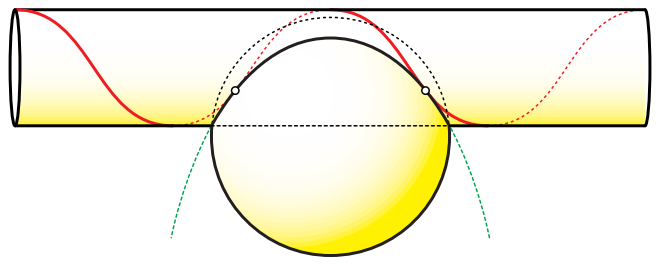

Figure 2. The intersection curve of the cylinder and a bitangent sphere projects to a parabola on the $x y$-plane.

The mean value theorem implies that $\gamma(x)=\cos x$ at most four times in the range $-\pi<x<\pi$. (Otherwise, the curves $y^{\prime \prime}=-\cos x$ and $y^{\prime \prime}=\gamma^{\prime \prime}(x)=1 / a$ would intersect more than twice in that range.) Since the curves meet with even multiplicity at two points, those are the only intersection points in the range $-\pi<x<$ $\pi$. Since $\gamma(x)$ is concave, we have $\gamma( \pm \pi)<\cos \pm \pi=$ -1 , so there are no intersections with $|x| \geq \pi$. Thus, the curves meet only at their two points of tangency.

Corollary 2.2. Any set $S$ of $n$ points on the helix $h(t)$ in the range $-\pi<t<\pi$ has a neighborly Delaunay triangulation.

Proof: Let $p$ and $q$ be arbitrary points in $S$, and let $\beta$ be the unique ball tangent to the helix at $p$ and $q$. By Lemma 2.1, $\beta$ does not otherwise intersect the helix and therefore contains no point in $S$. Thus, $p$ and $q$ are neighbors in the Delaunay triangulation of $S$.

We can now easily complete the analysis of our helical point set $S_{\sqrt{n}}$. Lemma 2.1 implies that every point in $S_{\sqrt{n}}$ is connected by a Delaunay edge to every other point less than a full turn around the helix $h \sqrt{1 / n}(t)$, and each full turn of the helix contains $\lfloor 2 \pi \sqrt{n}\rfloor$ points. Thus, the number of edges in the Delaunay triangulation of $S_{\sqrt{n}}$ is at least $2 \pi n^{3 / 2}-\Theta(n)$.
Theorem 2.3. For any $n$, there is a set of $n$ points in $\mathbb{R}^{3}$ with spread $\sqrt{n}$ whose Delaunay triangulation has complexity $\Omega\left(\mathrm{n}^{3 / 2}\right)$. Moreover, this point set is a uniform sample of a smooth convex surface with constant local feature size.

We can generalize our helix construction to other values of the spread $\Delta$ as follows.

Theorem 2.4. For any $\mathrm{n}$ and $\Delta=\Omega\left(\mathrm{n}^{1 / 3}\right)$, there is a set of $\mathrm{n}$ points in $\mathbb{R}^{3}$ with spread $\Delta$ whose Delaunay triangulation has complexity $\Omega\left(\min \left\{\Delta^{3}, \mathrm{n} \Delta, \mathrm{n}^{2}\right\}\right)$.

Proof: There are three cases to consider, depending on whether the spread is at least $n$, between $\sqrt{n}$ and $n$, or at most $\sqrt{n}$. The first case is trivial. For the case $\sqrt{n} \leq \Delta \leq n$, we take a set of evenly spaced points on a helix with pitch $\Delta / \mathrm{n}$ :

$$
\mathrm{S}_{\Delta}=\left\{\left(\frac{\mathrm{t}}{\mathrm{n}}, \cos \frac{\mathrm{t}}{\Delta}, \sin \frac{\mathrm{t}}{\Delta}\right) \mid \mathrm{t} \in[\mathrm{n}]\right\} .
$$

Every point in $S_{\Delta}$ is connected by a Delaunay edge to every other point less than a full turn away on the helix, and each turn of the helix contains $\Omega(\Delta)$ points, so the total complexity of the Delaunay triangulation is $\Omega(\mathrm{n} \Delta)$.

The final case $n^{1 / 3} \leq \Delta \leq \sqrt{n}$ is slightly more complicated. Our point set consists of several copies of our helix construction, with the helices positioned at the points of a square lattice, so the entire construction loosely resembles a mattress. Specifically, $S_{\Delta}$ is the set $\left\{\left(\frac{t}{r}, 4 i+\cos \frac{t}{\sqrt{r}}, 4 j+\sin \frac{t}{\sqrt{r}}\right) \mid t \in[w r] ; i, j \in[w]\right\}$, where $r$ and $w$ are parameters to be determined shortly. This set contains $n=w^{3} r$ points. The diameter of $S_{\Delta}$ is $\Theta(w)$ and the closest pair distance is $\Theta(1 / \sqrt{r})$, so its spread is $\Delta=\Theta(w \sqrt{r})$. Thus, given $n$ and $\Delta$, we have $w=\Theta\left(n / \Delta^{2}\right)$ and $r=\Theta\left(\Delta^{6} / n^{2}\right)$. Straightforward calculations imply that for all $t<\pi / 4$ and $\alpha<1$, the bitangent sphere $\beta_{\alpha}(t)$ has radius less than 2 . Since adjacent helices are separated by distance 2 , every point in $S_{\Delta}$ is connected in the Delunay triangulation to every point at most half a turn away in the same helix. Each turn of each helix contains $\Omega(\sqrt{r})$ points, so the Delaunay triangulation of $S_{\Delta}$ has complexity $\Omega(n \sqrt{r})=\Omega\left(\Delta^{3}\right)$.

\subsection{Upper Bounds}

Let $B$ be a ball of radius $R$ in $\mathbb{R}^{3}$, and let $b_{1}, b_{2}, b_{3}, \ldots$ be balls of radius at least $r$, where $1 \leq r \leq R$. Our upper bound proof uses the following geometric properties of the 'Swiss cheese' $C=B \backslash \bigcup_{i} b_{i}$. See Figure 3. 


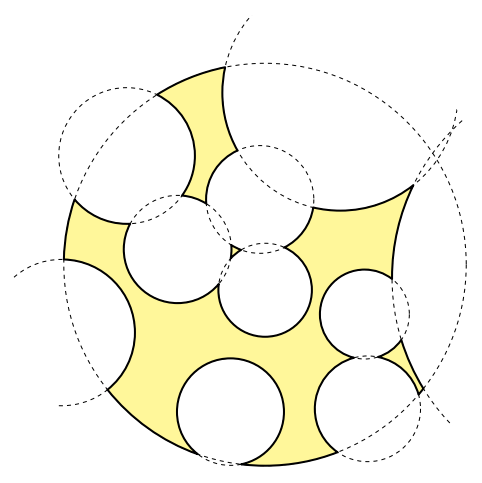

Figure 3. Swiss cheese (in $\mathbb{R}^{2}$ )

Lemma 2.5. The surface area of $\mathrm{C}$ is $\mathrm{O}\left(\mathrm{R}^{3} / \mathrm{r}\right)$.

Proof: The outer surface $\partial C \cap \partial B$ clearly has area $O\left(R^{2}\right)=O\left(R^{3} / r\right)$, so it suffices to bound the surface area of the 'holes'. For each $i$, let $H_{i}=B \cap \partial b_{i}$ be the boundary of the ith hole, and let $H=\bigcup_{i} H_{i}=\partial C \backslash \partial B$. For any point $x \in H$, let $s_{x}$ denote the open line segment of length $r$ extending from $x$ towards the center of the ball $b_{i}$ with $x$ on its boundary. (If $x$ lies on the surface of more than one $b_{i}$, choose one arbitrarily.) Let $S=\bigcup_{x \in H} s_{x}$ be the union of all such segments, and for each $i$, let $S_{i}=\bigcup_{x \in H_{i}} s_{x}$. Each $S_{i}$ is a fragment of a spherical shell of thickness $r$ inside the ball $b_{i}$. See Figure 4.

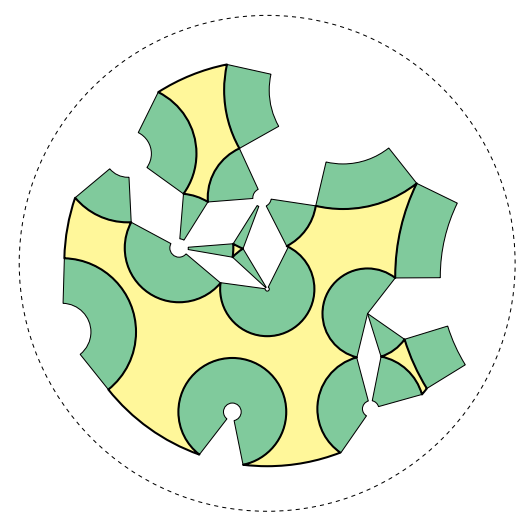

Figure 4. Shell fragments used to bound the surface area of C.

For each $i$, we have (after some tedious calculations)

$$
\operatorname{vol}\left(S_{i}\right)=\left(\frac{r_{i}}{3}-\frac{\left(r-r_{i}\right)^{3}}{3 r_{i}^{2}}\right) \operatorname{area}\left(H_{i}\right) \geq \frac{r}{3} \operatorname{area}\left(H_{i}\right),
$$

where $r_{i} \geq r$ is the radius of $b_{i}$. The triangle inequality implies that $s_{x}$ and $s_{y}$ are disjoint for any two points $x, y \in H$, so the shell fragments $S_{i}$ are pairwise disjoint. Finally, since $S$ fits inside a ball of radius $R+r \leq 2 R$, its volume is $\mathrm{O}\left(\mathrm{R}^{3}\right)$. Thus, $\operatorname{area}(\mathrm{H})=\sum_{i} \operatorname{area}\left(H_{i}\right) \leq$ $\sum_{i} 3 \operatorname{vol}\left(S_{i}\right) / r=3 \operatorname{vol}(S) / r=O\left(R^{3} / r\right)$.
Lemma 2.6. Let $\mathrm{U}$ be any unit ball whose center is in $\mathrm{C}$ and at distance $2 / 3$ from $\partial \mathrm{C}$. Then $\mathrm{U}$ contains $\Omega(1)$ surface area of $\mathrm{C}$.

Proof: Without loss of generality, assume that $U$ is centered at the origin and that $(0,0,2 / 3)$ is the closest point of $\partial C$ to the origin. Let $U^{\prime}$ be the open ball of radius $\delta$ centered at the origin, let $\mathrm{V}$ be the open unit ball centered at $(0,0,5 / 3)$, and let $W$ be the cone whose apex is the origin and whose base is the circle $\partial \mathrm{U} \cap \partial \mathrm{V}$. See Figure 5. $U^{\prime}$ lies entirely inside $C$, and since $r \geq 1$, we easily observe that $V$ lies entirely outside $C$. Thus, the surface area of $\partial \mathrm{C} \cap W \subseteq \partial \mathrm{C} \cap \mathrm{U}$ is at least the area of the spherical cap $\partial U^{\prime} \cap W$, which is exactly $4 \pi / 27$.

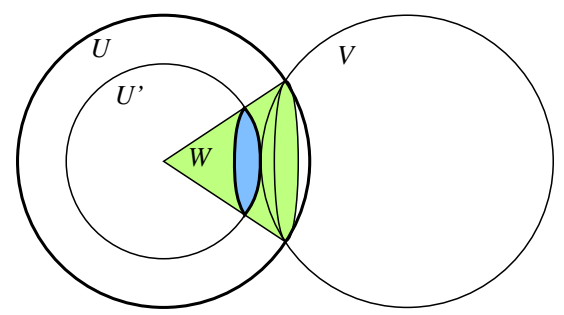

Figure 5. Proof of Lemma 2.6

Theorem 2.7. Let $S$ be a set of points in $\mathbb{R}^{3}$ whose closest pair is at distance 2, and let $r$ be any real number. Any point in $\mathrm{S}$ has $\mathrm{O}\left(\mathrm{r}^{2}\right)$ Delaunay neighbors at distance at most $\mathrm{r}$.

Proof: Let $\mathrm{o}$ be an arbitrary point in $S$, and let $B$ be a ball of radius $r$ centered at o. Call a Delaunay neighbor of $o$ a friend if it lies inside $B$, and call a friend $q$ interesting if there is another point $p \in S$ (not necessarily a Delaunay neighbor of o) such that $|o p|<$ $|\mathrm{oq}|$ and $\angle \mathrm{poq}<1 / \mathrm{r}$. A simple packing argument shows that o has at most $\mathrm{O}\left(\mathrm{r}^{2}\right)$ boring friends.

Let $Q$ be the set of interesting friends of o. Every point $q \in Q$ lies on the boundary of a Delaunay ball $d_{q}$ that contains no points of $S$ in its interior and also has o on its boundary. It is straightforward to prove that because $q$ is interesting and has distance at least 2 from any other point, $d_{q}$ must have radius at least $r$. Let $b_{q}$ be the ball concentric with $d_{q}$ with radius $2 / 3$ less than the radius of $\mathrm{d}_{\mathrm{q}}$. Finally, for any point $\mathrm{q}$, let $\mathrm{U}_{\mathrm{q}}$ be the unit-radius ball centered at $q$.

We now have a set of unit balls, one for each interesting friend of $\mathrm{o}$, whose centers lie at distance exactly $2 / 3$ from the boundary of the Swiss cheese $C=$ $\mathrm{B} \backslash \bigcup_{\mathrm{q} \in \mathrm{Q}} \mathrm{b}_{\mathrm{q}}$. By Lemma $2.5, \mathrm{C}$ has surface area $\mathrm{O}\left(\mathrm{r}^{2}\right)$, and by Lemma 2.6, each unit ball $\mathrm{u}_{\mathrm{q}}$ contains $\Omega(1)$ surface area of $C$. Since the unit balls are disjoint, it follows that o has at most $\mathrm{O}\left(\mathrm{r}^{2}\right)$ interesting friends. 
Theorem 2.8. Let $S$ be a set of points in $\mathbb{R}^{3}$ whose closest pair is at distance 2 and whose diameter is $2 \Delta$, and let $\mathrm{r}$ be any real number. There are $\mathrm{O}\left(\Delta^{3} / \mathrm{r}\right)$ points in $\mathrm{S}$ with a Delaunay neighbor at distance at least $\mathrm{r}$.

Proof: Call a point far-reaching if it has a Delaunay neighbor at distance at least $r$, and let $Q$ be the set of far-reaching points. Let $\mathrm{B}$ be a ball of radius $2 \Delta$ containing $S$. For each $q \in Q$, let $f_{q}$ be a maximal empty ball containing $\mathrm{q}$ and its furthest Delaunay neighbor, and let $b_{q}$ be the concentric ball with radius $2 / 3$ smaller than $f_{p}$. By construction, each ball $b_{q}$ has radius at least $r / 2-2 / 3$. Finally, for any far-reaching point $q$, let $U_{q}$ be the unit-radius ball centered at $q$. By Lemma 2.5, the Swiss cheese $C=B \backslash \bigcup_{q \in Q} b_{q}$ has surface area $\mathrm{O}\left(\Delta^{3} / \mathrm{r}\right)$, and by Lemma 2.6 , each unit ball $\mathrm{U}_{\mathrm{q}}$ contains $\Omega(1)$ surface area of $\mathrm{C}$. Since these unit balls are disjoint, there are at most $\mathrm{O}\left(\Delta^{3} / \mathrm{r}\right)$ of them.

Corollary 2.9. Let $S$ be a set of points in $\mathbb{R}^{3}$ with spread $\Delta$. The Delaunay triangulation of $S$ has complexity $\mathrm{O}\left(\Delta^{4}\right)$.

Proof: For all $r$, let $F(r)$ be the number of far-reaching points in $S$, i.e., those with Delaunay edges of length at least $r$. From Theorem 2.8, we have $\mathrm{F}(\mathrm{r})=\mathrm{O}\left(\Delta^{3} / \mathrm{r}\right)$. By Theorem 2.7, if the farthest neighbor of a point $p$ is at distance between $r$ and $r+1$, then $p$ has $O\left(r^{2}\right)$ neighbors. Thus, the total number of Delaunay edges is at most

$$
\begin{aligned}
\sum_{\mathrm{r}=0}^{\Delta} \mathrm{O}\left(\mathrm{r}^{2}\right) \cdot(\mathrm{F}(\mathrm{r})-\mathrm{F}(\mathrm{r}+1)) & =\sum_{\mathrm{r}=0}^{\Delta} \mathrm{O}(\mathrm{r}) \cdot \mathrm{F}(\mathrm{r}) \\
& =\sum_{\mathrm{r}=0}^{\Delta} \mathrm{O}\left(\Delta^{3}\right) \\
& =\mathrm{O}\left(\Delta^{4}\right)
\end{aligned}
$$

\subsection{Conjectured Upper Bounds}

I conjecture that the lower bounds in Theorem 2.4 are tight, but Corollary 2.9 is the best upper bound known. Nearly matching upper bounds could be derived from the following conjecture using a divide and conquer argument, suggested by Edgar Ramos (personal communication).

Let $S$ be a well-separated set of points with closest pair distance 1, lying in two balls of radius $\Delta$ that are separated by distance at least $\mathrm{c} \Delta$ for some constant $c>1$. Call an edge in the Delaunay triangulation of $S$ a crossing edge if it has one endpoint in each ball.
Conjecture 2.10. Some point in $S$ is an endpoint of $\mathrm{O}(\Delta)$ crossing edges.

Lemma 2.11. Conjecture 2.10 implies that the Delaunay triangulation of $\mathrm{S}$ has $\mathrm{O}\left(\min \left\{\Delta^{3}, \Delta \mathrm{n}, \mathrm{n}^{2}\right\}\right)$ crossing edges.

Proof: Theorem 2.8 implies that only $\mathrm{O}\left(\Delta^{2}\right)$ points can be endpoints of crossing edges. Thus, we can assume without loss of generality that $\mathrm{n}=\mathrm{O}\left(\Delta^{2}\right)$.

We compute the total number of crossing edges by iteratively removing the point with the fewest crossing edges and retriangulating the resulting hole, say by incremental flipping. Conjecture 2.10 implies that we delete only $\mathrm{O}(\Delta)$ crossing edges with each point, so altogether we delete $\mathrm{O}(\mathrm{n} \Delta)=\mathrm{O}\left(\Delta^{3}\right)$ crossing edges. Not all of these edges are in the original Delaunay triangulation, but that only helps us.

Theorem 2.12. Conjecture 2.10 implies that the Delaunay triangulation of $n$ points in $\mathbb{R}^{3}$ with spread $\Delta$ has complexity $\mathrm{O}\left(\min \left\{\Delta^{3} \log \Delta, \mathrm{n} \Delta, \mathrm{n}^{2}\right\}\right)$.

Proof: Assume Conjecture 2.10 is true, and let $S$ be an arbitrary set of $n$ points with diameter $\Delta$, where the closest pair of points is at unit distance. $S$ is contained in an axis-parallel cube $C$ of width $\Delta$. We construct a well-separated pair decomposition of $S$ [13], based on a simple octtree decomposition of $\mathrm{C}$. The octtree has $\mathrm{O}(\log \Delta)$ levels. At each level $i$, there are $8^{i}$ cells, each a cube of width $\Delta / 2^{i}$. Our well-separated pair decomposition includes, for each level $i$, the points in any pair of level- $i$ cells separated by a distance between $c \Delta / 2^{i}$ and $2 c \Delta / 2^{i}$. A simple packing argument implies that any cell in the octtree is paired with $\mathrm{O}(1)$ other cells, all at the same level, and so any point appears in $\mathrm{O}(\log \Delta)$ subset pairs. Every Delaunay edge of $\mathrm{S}$ is a crossing edge for some well-separated pair of cells.

Lemma 2.11 implies that the points in any wellseparated pair of level-i cells have $\mathrm{O}\left(\Delta^{3} / 8^{i}\right)$ crossing Delaunay edges. Since there are $\mathrm{O}\left(8^{i}\right)$ such pairs, the total number of crossing edges between level- $i$ cells is $\mathrm{O}\left(\Delta^{3}\right)$. Thus, there are $\mathrm{O}\left(\Delta^{3} \log \Delta\right)$ Delaunay edges altogether.

Lemma 2.11 also implies that for any well-separated pair of level- $i$ cells, the average number of crossing edges per point is $\mathrm{O}\left(\Delta / 2^{i}\right)$. Since every point belongs to a constant number of subset pairs at each level, the total number of crossing edges at level $i$ is $\mathrm{O}\left(\mathrm{n} \Delta / 2^{i}\right)$. Thus, the total number of Delaunay edges is $\mathrm{O}(\mathrm{n} \Delta)$.

This upper bound is still a logarithmic factor away from our lower bound construction when $\Delta=\mathrm{o}(\sqrt{n})$. 
However, our argument is quite conservative; all crossing edges for a well-separated pair of subsets are counted, even though some or all of these edges may be blocked by other points in S. A more careful analysis would probably eliminate the final logarithmic factor.

\section{Nice Surface Data}

Let $\Sigma$ be a smooth surface without boundary in $\mathbb{R}^{3}$. The medial axis of $\Sigma$ is the closure of the set of points in $\mathbb{R}^{3}$ that have more than one nearest neighbor on $\Sigma$. The local feature size of a point $x \in \Sigma$, denoted lfs $(x)$, is the distance from $x$ to the medial axis of $\Sigma$. Let $S$ be a set of sample points on $\Sigma$. Following Amenta and Bern [1], we say that $S$ is an $\varepsilon$-sample of $\Sigma$ if the distance from any point $x \in \Sigma$ to the nearest sample point is at most $\varepsilon \cdot \operatorname{lfs}(x)$.

The first step in several surface reconstruction algorithms is to construct the Delaunay triangulation or Voronoi diagram of the sample points. Edelsbrunner and Mücke [22] and Bajaj et al. [7, 9] describe algorithms based on alpha shapes, which are subcomplexes of the Delaunay triangulation; see also [25]. Extending earlier work on planar curve reconstruction [2, 23], Amenta and Bern [1,3] developed an algorithm to extract a certain manifold subcomplex of the Delaunay triangulation, called the crust. Amenta et al. [4] simplified the crust algorithm and proved that if $S$ is an $\varepsilon$-sample of a smooth surface $\Sigma$, for some sufficiently small $\varepsilon$, then the crust is homeomorphic to $\Sigma$. Boissonnat and Cazals [12] and Hiyoshi and Sugihara [26] proposed algorithms to produce a smooth surface using natural coordinates, which are defined and computed using the Voronoi diagram of the sample points. Further examples can be found in $[5,6,11,15]$.

In this section, we show that $\varepsilon$-samples of smooth surfaces can have complicated Delaunay triangulations, implying that all these surface reconstruction algorithms can take quadratic time in the worst case. We will analyze our constructions in terms of the sample measure of a surface $\Sigma$, which we define as follows:

$$
\mu(\Sigma)=\int_{\Sigma} \frac{\mathrm{d} x}{\operatorname{lfs}^{2}(x)} .
$$

Lemma 3.1. For all $\varepsilon<1 / 2$, every $\varepsilon$-sample of $\Sigma$ contains $\Omega\left(\mu(\Sigma) / \varepsilon^{2}\right)$ points.

Proof: Let $S$ be an arbitrary $\varepsilon$-sample of $\Sigma$. Amenta and Bern [1] observed that $|\mathrm{lfs}(\mathrm{p})-\mathrm{lfs}(\mathrm{q})|<|\mathrm{pq}|$ for any points $p, q \in \Sigma$. This observation implies that for any point $x \in \Sigma$, we have $|x p| \leq \varepsilon \operatorname{lfs}(x) \leq$ $\varepsilon(\operatorname{lfs}(p)+|x p|) \leq \frac{\varepsilon}{1-\varepsilon} \operatorname{lfs}(p)$, where $p \in S$ is the sample point closest to $x$. Thus, we can cover $\Sigma$ with circular neighborhoods of radius $\frac{\varepsilon}{1-\varepsilon} \operatorname{lfs}(\mathrm{p})$ around each sample point $p \in S$. By similar arguments, the neighborhood of $p$ has area at least $\pi\left(\frac{\varepsilon}{1-\varepsilon}\left(1-\frac{\varepsilon}{1-\varepsilon}\right) \operatorname{lfs}(p)\right)^{2}$, and any point in the neighborhood of $p$ has local feature size at most $\left(1+\frac{\varepsilon}{1-\varepsilon}\right) \operatorname{lfs}(p)$. It follows that each neighborhood has sample measure $\Omega\left(\varepsilon^{2}\right)$, and since there are $n$ such neighborhoods, $\mu(\Sigma)=\mathrm{O}\left(\mathrm{n} \varepsilon^{2}\right)$.

We say that an $\varepsilon$-sample is parsimonious if it contains $\mathrm{O}\left(\mu(\Sigma) / \varepsilon^{2}\right)$ points.

\subsection{Oversampling Is Bad}

The easiest method to produce a surface sample with high Delaunay complexity is oversampling, where some region of the surface contains many more points than necessary. In fact, the only surface where oversampling cannot produce a quadratic-complexity Delaunay triangulation is the sphere, even if we only consider parsimonious samples.

Theorem 3.2. For any smooth non-spherical surface $\Sigma$, any $\varepsilon>0$, and any sufficiently large $\mathrm{n}$, there is a parsimonious $\varepsilon$-sample of $\Sigma$ of size $n$ whose Delaunay triangulation has complexity $\Omega\left(\mathrm{n}^{2}\right)$.

Proof: Let $S$ be any parsimonious $\varepsilon$-sample of $\Sigma$. Let $\sigma$ be a small sphere intersecting $\Sigma$ in a non-planar curve, where the distance from $\sigma$ to any point os $S$ is at elast the radius of $\sigma$. Such a sphere always exists unless $\Sigma$ is itself a sphere. Let $\alpha$ and $\beta$ be extremely short segments of the intersection curve $\Sigma \cap \sigma$ that approximate skew line segments. Straighten these curves slightly, keeping them on the surface $\Sigma$ and keeping the endpoints fixed, to obtain curves $\alpha^{\prime}$ and $\beta^{\prime}$. Finally, let $A$ and B be sets of $|S|$ evenly spaced points on $\alpha^{\prime}$ and $\beta^{\prime}$, respectively. See Figure 6.

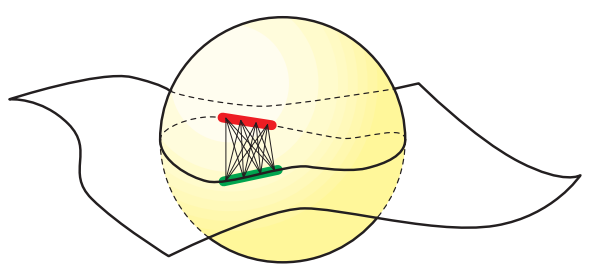

Figure 6. Parsimoniously oversampling a non-spherical surface.

The Delaunay triangulation of $A \cup B$ has complexity $\Omega\left(|S|^{2}\right)$; every point in $A$ is a Delaunay neighbor of every point in B. Moreover, any Delaunay circumsphere of $A \cup B$ closely approximates the sphere $\sigma$ and thus 
excludes every point in $S$. Thus, $S \cup A \cup B$ is a parsimonious $\varepsilon$-sample of $\Sigma$ consisting of $n=3|S|$ points whose Delaunay triangulation has complexity $\Omega\left(n^{2}\right)$.

The reconstruction algorithm of Amenta et al. [4] extracts a surface from a subset of the Delaunay triangles of the sample points. Their algorithm estimates the surface normal at each smple point $p$ using the Voronoi diagram of the samples. The cocone at $p$ is the complement of a very wide double cone whose apex is $p$ and whose axis is the estimated normal vector at $p$. The algorithm extracts the Delaunay triangles whose dual Delaunay edges intersect the cocones of all three of its vertices, and then extracts a manifold surface from those cocone triangles. Usually only a small subset of the Delaunay triangles pass this filtering phase, but our construction shows that there can be $\Omega\left(n^{2}\right)$ cocone triangles in the worst case.

\subsection{Uniform Sampling Can Still Be Bad}

Unfortunately, oversampling is not the only way to get quadratic Delaunay triangulations. Let $S$ be a set of sample points on the surface $\Sigma$. We define the second sampling density of a point $x \in \Sigma$, denoted $\operatorname{sd}_{2}(x)$, as the distance from $x$ to the second closest sample point, divided by $\operatorname{lfs}_{\mathrm{s}}(\mathrm{x})$. We say that $S$ is a uniform $\varepsilon$-sample of $\Sigma$ if $\varepsilon / 4 \leq \operatorname{sd}_{2}(x) \leq \varepsilon$ for all $x \in \Sigma{ }^{1}$ Uniform $\varepsilon$-samples are also parsimonious $\varepsilon$-samples, but with absolutely no oversampling. In particular, the size of any uniform $\varepsilon$-sample is $\Theta\left(\mu(\Sigma) / \varepsilon^{2}\right)$.

Lemma 3.3. For any $n$ and $\varepsilon>\sqrt{1 / n}$, there is a twocomponent surface $\Sigma$ and an n-point uniform $\varepsilon$-sample $\mathrm{S}$ of $\Sigma$, such that the Delaunay triangulation of $S$ has complexity $\Omega\left(n^{2} \varepsilon^{2}\right)$.

Proof: The surface $\Sigma$ is the boundary of two sausages $\Sigma_{x}$ and $\Sigma_{y}$, each of which is the Minkowski sum of a unit sphere and a line segment. Specifically, let

$$
\begin{aligned}
& \Sigma_{x}=\mathrm{U}+\overline{(-w, 0, \mathrm{~d}+1),(w, 0, \mathrm{~d}+1)} \quad \text { and } \\
& \Sigma_{y}=\mathrm{U}+\overline{(0,-w,-\mathrm{d}-1),(0, w,-\mathrm{d}-1)},
\end{aligned}
$$

where $U$ is the unit ball centered at the origin, $w=n \varepsilon^{2}$, and $d=4 w / \varepsilon=4 n \varepsilon$. The local feature size of every point on $\Sigma$ is 1 , so any uniform $\varepsilon$-sample of $\Sigma$ has $\Theta\left((w+1) / \varepsilon^{2}\right)=\Theta(n)$ points.

\footnotetext{
${ }^{1}$ There is nothing special about the number 4 here; any constant $c>2$ will do. However, as $c$ approaches 2 , the maximum $\varepsilon$ for which a $c$-uniform $\varepsilon$-sample exists approaches zero.
}

Define the seams $\sigma_{x}$ and $\sigma_{y}$ as the maximal line segments in each sausage closest to the $x y$-plane:

$$
\begin{aligned}
\sigma_{x} & =\overline{(-w, 0, d),(w, 0, d)} \quad \text { and } \\
\sigma_{y} & =\overline{(0,-w,-d),(0, w,-d)} .
\end{aligned}
$$

Our uniform $\varepsilon$-sample $S$ contains $2 w / \varepsilon+1$ points along each seam:

$p_{i}=(i \varepsilon, 0, d) \quad$ for all integers $-w / \varepsilon \leq i \leq w / \varepsilon$, and

$q_{j}=(0, j \varepsilon,-d)$ for all integers $-w / \varepsilon \leq j \leq w / \varepsilon$.

The Delaunay triangulation of these $\Theta(w / \varepsilon)=\Theta(n \varepsilon)$ points has complexity $\Theta\left(w^{2} / \varepsilon^{2}\right)=\Theta\left(n^{2} \varepsilon^{2}\right)$.

Let $\gamma_{i j}$ be the ball whose boundary passes through $p_{i}$ and $q_{j}$ and is tangent to both seams. This ball may contain other portions of the surface, but we claim that the intersection is small enough that we can avoid it with our sample points. The intersection of $\Sigma_{x}$ and $\gamma_{i j}$ is a small oval, tangent to $p_{i}$ and symmetric about the plane $x=i \varepsilon$. Tedious calculation (which we omit) implies that the width of the oval is

$$
2 \tan ^{-1}\left(\frac{4 d j \varepsilon}{4 d(d+1)+\left(i^{2}-j^{2}\right) \varepsilon^{2}}\right)<\frac{4 w}{d}=\varepsilon .
$$

See Figure 7.

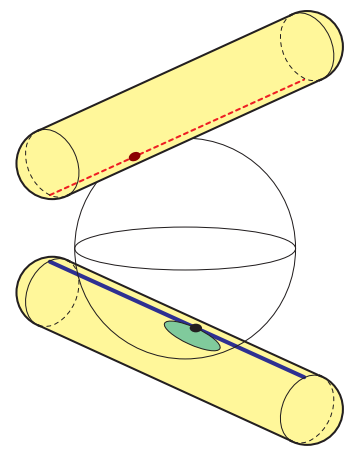

Figure 7. Two sausages and a sphere tangent to both seams.

So $\Sigma_{x} \cap \gamma_{i j}$ lies entirely within a strip of width $2 \varepsilon$ centered along the seam $\sigma_{x}$. A symmetric argument gives the analogous result for $\Sigma_{y} \cap \gamma_{i j}$. We can uniformly sample $\Sigma$ so that no other sample point lies within either strip. Each segment $\overline{p_{i} q_{j}}$ is an edge in the Delaunay triangulation of the sample, and there are $\Omega\left(w^{2} / \varepsilon^{2}\right)=$ $\Omega\left(n^{2} \varepsilon^{2}\right)$ such segments.

Theorem 3.4. For any $n$ and any $\varepsilon>\sqrt{(\log n) / n}$, there is a connected surface $\Sigma$ and an n-point uniform $\varepsilon$-sample $S$ of $\Sigma$, such that the Delaunay triangulation of $S$ has complexity $\Omega\left(n^{2} \varepsilon^{2}\right)$. 
Proof: Intuitively, we produce the surface $\Sigma$ by pushing two sausages into a spherical balloon. These sausages create a pair of conical wedges inside the balloon whose seams lie along two skew lines. The local feature size is small near the seams and drops off quickly elsewhere, so a large fraction of the points in any uniform sample must lie near the seams. We construct a particular sample with points exactly along the seams that form a quadratic-complexity triangulation, similarly to our earlier sausage construction. Our construction relies on several parameters: the radius $R$ of the spherical balloon, the width $w$ and height $h$ of the wedges, and the distance $d$ between the seams.

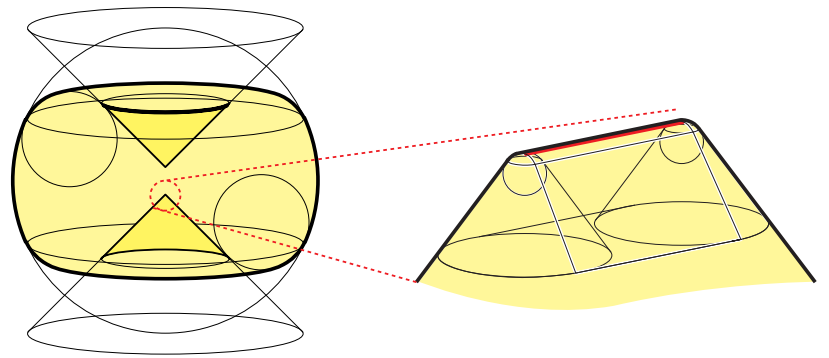

Figure 8. A smooth surface with a bad uniform $\varepsilon$-sample, and a closeup of one of its wedges.

Each wedge is the Minkowski sum of a unit sphere, a right circular cone with height $h$ centered along the $z$-axis, and a line segment of length $w$ parallel to one of the other coordinate axes. The boundary of each wedge can be decomposed into cylindrical, spherical, conical, and planar facets. The cylindrical and spherical facets constitute the blade of the wedge, and the seam of the blade is the line segment of length $w$ that bisects the cylindrical facet. The local feature size of any point on the blade is exactly 1 , and the local feature size of any other boundary point is its distance from the blade. Straightforward calculations imply that the sample measure of the wedge is $\mathrm{O}(w+\log h+1)$.

A first approximation $\widetilde{\Sigma}$ of the surface $\Sigma$ is obtained by removing two wedges from a ball of radius $R$ centered at the origin. One wedge points into the ball from below; its seam is parallel to the $x$-axis and is centered at the point $(0,0,-R+h)$. The other wedge points into the ball from above; its seam is parallel to the $y$-axis and is centered at $(0,0, R-h)$. Let $d=2 R-2 h-2$ denote the distance between the wedges. Our construction has $1 \ll w \ll d \ll h$, so $R<3 h$.

To obtain the final smooth surface $\Sigma$, we round off the sharp edges by rolling a ball of radius $h / 4$ inside $\widetilde{\Sigma}$ along the wedge/balloon intersection curves. We call the resulting warped toroidal patches the sleeves. The local feature size of any point on the sleeves or on the balloon is at least $h / 4$. Since $\Sigma$ is star-shaped and contained in a sphere of radius $R$, its surface area is at most $4 \pi R^{2}<36 \pi h^{2}$. It follows that the sleeves have constant sample measure. The local feature size of wedge points changes only far from the blades and by only a small constant factor, so $\mu(\Sigma)=\Theta(w+\log h+1)$. To complete the construction, we set $w=n \varepsilon^{2}, d=4 n \varepsilon$, and $h=20 n \varepsilon$. See Figure 8 .

Finally, we construct a uniform $\varepsilon$-sample $S$ with $\Theta(w / \varepsilon)$ sample points evenly spaced along each seam and every other point at least $\varepsilon$ away from the seams. Setting $h>5 d$ (and thus $R>10 d$ ) ensures that the Delaunay spheres $\gamma_{i j}$ between seam points do not touch the surface except on the blades. By the argument in Lemma 3.3, there are $\Omega\left(w^{2} / \varepsilon^{2}\right)=\Omega\left(n^{2} \varepsilon^{2}\right)$ Delaunay edges between seam points.

\subsection{Some Surfaces Are Just Evil}

In this section, we describe a family of surfaces for which any parsimonious $\varepsilon$-sample has a Delaunay triangulation of near-quadratic complexity. First we give a nearly trivial construction of a bad surface with several components, and then we join these components into a single connected surface using a method similar to Theorem 3.4.

Lemma 3.5. For any $n$ and any $\varepsilon<\sqrt{1 / n}$, there is a smooth surface $\Sigma$ such that the Delaunay triangulation of any parsimonious $\varepsilon$-sample of $\Sigma$ has complexity $\Omega\left(n^{2} \varepsilon^{4}\right)$, where $n$ is the size of the sample.

Proof: Let $\mathrm{P}$ be a set containing the following $\mathrm{k}$ points:

$p_{i}=\left(i k, 0, k^{2}\right) \quad$ for all integers $-k / 4 \leq i \leq k / 4$, and

$q_{j}=\left(0, j k,-k^{2}\right)$ for all integers $-k / 4 \leq j \leq k / 4$.

We easily verify that every pair of points $p_{i}$ and $q_{j}$ lie on a sphere $\gamma_{i j}$ with every other point in $P$ at least unit distance outside.

Let $\Sigma=\bigcup_{p \in P} U_{p}$, where $U_{p}$ is the unit-radius sphere centered at $p$. Clearly, lfs $(x)=1$ for every point $x \in \Sigma$, so $\mu(\Sigma)=4 \pi \mathrm{k}$. Let $S$ be an arbitrary parsimonious $\varepsilon$ sample of $\Sigma$, let $n=|S|=\Theta\left(k / \varepsilon^{2}\right)$, and for any point $p \in P$, let $S_{p}=S \cap U_{p}$ be the sample points on its unit sphere.

Choose an arbitrary Delaunay pair $p_{i}, q_{j} \in P$, and let $\gamma$ be a sphere concentric with $\gamma_{i j}$ but with radius smaller by 1 . This sphere is tangent to $U_{p_{i}}$ and $U_{q_{i}}$ but is at least unit distance from every other component of $\Sigma$. Expand $\gamma$ about its center until it hits (without loss of generality) a point $p^{\prime} \in S_{p_{i}}$, and then expand it 
about $p^{\prime}$ until it hits a point $q^{\prime} \in S_{p_{i}}$. The resulting sphere $\gamma$ passes through $p^{\prime}$ and $q^{\prime}$ and has no points of $S$ in its interior, so $p^{\prime}$ and $q^{\prime}$ are joined by an edge in the Delaunay triangulation of $S$. There are at least $\Omega\left(k^{2}\right)=\Omega\left(n^{2} \varepsilon^{4}\right)$ such edges.

To create a connected surface where good sample has a complicated Delaunay triangulation, we add 'teeth' to our earlier balloon and wedge construction. Unfortunately, in the process, we lose a logarithmic factor in the Delaunay complexity.

Theorem 3.6. For any $\mathrm{n}$ and any $\varepsilon<\sqrt{(\log n) / n}$, there is a smooth connected surface $\Sigma$ such that the Delaunay triangulation of any parsimonious $\varepsilon$-sample of $\Sigma$ has complexity $\Omega\left(n^{2} \varepsilon^{4} / \log ^{2}\left(n \varepsilon^{2}\right)\right)$, where $n$ is the size of the sample.

Proof: Intuitively, we create the surface $\Sigma$ by pushing two rows of regularly spaced unit balls into a large spherical balloon, similarly to the proof of Theorem 3.4. As before, the surface contains two wedges, but now each wedge has a row of small conical teeth. Our construction relies on the same parameters $R, w, h$ of our earlier construction. We now have additional parameter $t$, which is simultaneously the height of the teeth, the distance between the teeth, and half the thickness of the 'blade' of the wedge.

Our construction starts with the (toothless) surface described in the proof of Theorem 3.4, but using a ball of radius $t$ instead of a unit ball to define the wedges. We add $w / t$ evenly-spaced teeth along the blade of each wedge, where each tooth is the Minkowski sum of a unit ball with a right circular cone of radius $t$. Each tooth is tangent to both planar facets of its wedge. To create the final smooth surface $\Sigma$, we roll a ball of radius $t / 3$ over the blade/tooth intersection curves. The complete surface has sample measure $\Theta((w / t)(1+\log t)+\log h+1)$. Finally, we set the parameters $w=t^{2}, h=t^{3}$, and $R=20 t^{3}$, so that $\mu(\Sigma)=\Theta(t \log t)$.

Let $S$ be a parsimonious $\varepsilon$-sample of $\Sigma$, and let $n=$ $|S|=\Theta\left((t \log t) / \varepsilon^{2}\right)$. For any pair of teeth, one on each wedge, there is a sphere tangent to the ends of the teeth that has distance $\Omega(1)$ from the rest of the surface. We can expand this sphere so that it passes through one point on each tooth and excludes the rest of the points. Thus, the Delaunay triangulation of $S$ has complexity $\Omega\left(t^{2}\right)=\Omega\left(n^{2} \varepsilon^{4} / \log ^{2}\left(n \varepsilon^{2}\right)\right)$.

\subsection{Randomness Doesn't Help Much}

Golin and $\mathrm{Na}$ recently proved that if $\mathrm{S}$ is a random set of $n$ points on the surface of a convex polytope, then the expected complexity of the Delaunay triangulation of $S$ is $\mathrm{O}(n)$ [24]. Unfortunately, this result does not extend to nonconvex objects, even the random distribution of the points is proportional to the sample measure.

Theorem 3.7. For any $\mathrm{n}$, there is a smooth connected surface $\Sigma$, such that the Delaunay triangulation of $n$ independent uniformly-distributed random points in $\Sigma$ has complexity $\Theta\left(n^{2} / \log ^{2} n\right)$ with high probability.

Proof: Consider the surface $\Sigma$ consisting of $\Theta(n / \log n)$ unit balls evenly spaced along two skew line segments, exactly as in the proof of Theorem 3.5, with thin cylinders joining them into a single connected surface. With high probability, a random sample of $n$ points contains at least one point on each ball, on the side facing the opposite segment. Thus, with high probability, there is at least one Delaunay edge between any ball on one segment and any ball on the other segment.

Theorem 3.8. For any $\mathrm{n}$, there is a smooth connected surface $\Sigma$, such that the Delaunay triangulation of $\mathrm{n}$ independent random points in $\Sigma$, distributed proportionally to the sample measure, has complexity $\Theta\left(n^{2} / \log ^{4} n\right)$ with high probability.

Proof: Let $\Sigma$ be the surface used to prove Theorem 3.6, but with $\Theta\left(n / \log ^{2} n\right)$ teeth. With high probability, a weighted random sample of $\Sigma$ contains at least one point at the tip of each tooth.

\section{Conclusions}

We have derived new upper and lower bounds on the complexity of Delaunay triangulations under two different geometric constraints: point sets with sublinear spread and good samples of smooth surfaces. Our results imply that with very strong restrictions on the inputs, existing surface reconstruction algorithms are inefficient in the worst case.

Our results suggest several open problems, the most obvious of which is to tighten the spread-based bounds. Even the special case $\Delta=\Theta\left(n^{1 / 3}\right)$ is open.

Another natural open problem is to generalize our analysis to higher dimensions. Using the proof techniques in Section 2.2, we can show that any d-dimensional Delaunay triangulation has $\mathrm{O}\left(\Delta^{\mathrm{d}+1}\right)$ edges. We conjecture that the total complexity is 
always $\mathrm{O}\left(\Delta^{\mathrm{d}}\right)$ and can only reach the maximum $\Omega\left(n^{\lceil\mathrm{d} / 2\rceil}\right)$ when $\Delta=\Omega(\mathrm{n})$.

Our bad surface examples are admittedly contrived, since they have areas of very high curvature relative to their diameter. An interesting open problem is whether there are bad surfaces with smaller 'spread', i.e., ratio between diameter and minimum local feature size. What is the worst-case complexity of the Delaunay triangulation of good surface as a function of the spread and sample measure of the surface?

Our results imply that any Delaunay-based surface reconstruction algorithm can be forced to take superlinear time, even for very natural surface data. It may be possible to improve these algorithms by adding a small number of Steiner points in a preprocessing phase to reduce the complexity of the Delaunay triangulation. In most of our bad surface examples, a single Steiner point reduces the Delaunay complexity to $O(n)$. Bern, Eppstein, and Gilbert [10] show that any Delaunay triangulation can be reduced to $\mathrm{O}(n)$ complexity in $\mathrm{O}(n \log n)$ time by adding $\mathrm{O}(n)$ Steiner points; see also [14]. Unfortunately, the Steiner points they choose (the vertices of an octtree) may make reconstruction impossible. In order to be usable, any new Steiner points must either lie very close to or very far from the surface, and as our bad examples demonstrate, both types of Steiner points may be necessary. Boissonnat and Cazals (personal communication) report that adding a small subset of the original Voronoi vertices as Steiner points can significantly reduce the complexity of the resulting Voronoi diagram with only minimal changes to the smooth surface constructed by their algorithm [12].

Very recently, Dey et al. [18] developed a surface reconstruction algorithm that does not construct the entire Delaunay triangulation. Their algorithm runs in $\mathrm{O}(n \log n)$ time if (loosely speaking) the density of the sample points varies smoothly over the surface.

Finally, are there other natural geometric conditions under which the Delaunay triangulation provably has small complexity?

Acknowledgments. I thank Herbert Edelsbrunner for asking the (still open!) question that started this work, Kim Whittlesey for suggesting charging Delaunay features to area, and Edgar Ramos for suggesting wellseparated pair decompositions and sending me a copy of his paper [18]. Thanks also to Sariel Har-Peled, Olivier Devillers, and Jean-Daniel Boissonnat for helpful discussions.

\section{References}

[1] N. Amenta and M. Bern. Surface reconstruction by Voronoi filtering. Discrete Comput. Geom. 22(4):481-504, 1999.

[2] N. Amenta, M. Bern, and D. Eppstein. The crust and the $\beta$-skeleton: Combinatorial curve reconstruction. Graph. Models Image Process. 60:125-135, 1998.

[3] N. Amenta, M. Bern, and M. Kamvysselis. A new Voronoi-based surface reconstruction algorithm. Proc. SIGGRAPH '98, 415-412, 1998.

[4] N. Amenta, S. Choi, T. K. Dey, and N. Leekha. A simple algorithm for homeomorphic surface reconstruction. Proc. 16th Annu. ACM Sympos. Comput. Geom., 213-222, 2000.

[5] N. Amenta, S. Choi, and R. Kolluri. The power crust, unions of balls, and the medial axis transform. To appear in Internat. J. Comput. Geom. Appl. /http://www.cs.utexas.edu/users/amenta/ pubs/power.ps.gz $\rangle$.

[6] D. Attali. r-regular shape reconstruction from unorganized points. Comput. Geom. Theory Appl. 10:239-247, 1998.

[7] C. Bajaj, F. Bernardini, and G. Xu. Automatic reconstruction of surface and scalar fields from $3 \mathrm{~d}$ scans. Comput. Graph. 29:109-118, 1995. Proc. SIGGRAPH '95.

[8] M. de Berg, M. J. Katz, A. F. van der Stappen, and J. Vleugels. Realistic input models for geometric algorithms. Proc. 13th Annu. ACM Sympos. Comput. Geom., 294-303, 1997.

[9] F. Bernardini and C. L. Bajaj. Sampling and reconstructing manifolds using alpha-shapes. Proc. 9th Canad. Conf. Comput. Geom., 193-198. 1997.

[10] M. Bern, D. Eppstein, and J. Gilbert. Provably good mesh generation. J. Comput. Syst. Sci. 48:384-409, 1994.

[11] J.-D. Boissonnat. Representing $2 d$ and $3 d$ shapes with the Delaunay triangulation. Proc. 7th IEEE Internat. Conf. Pattern Recogn., 745-748, 1984.

[12] J.-D. Boissonnat and F. Cazals. Smooth surface reconstruction via natural neighbour interpolation of distance functions. Proc. 16th Annu. ACM Sympos. Comput. Geom., 223-232, 2000.

[13] P. B. Callahan and S. R. Kosaraju. A decomposition of multidimensional point sets with applications to k-nearest-neighbors and n-body potential fields. J. ACM 42:67-90, 1995.

[14] B. Chazelle, H. Edelsbrunner, L. Guibas, J. Hershberger, R. Seidel, and M. Sharir. Selecting heavily 
covered points. SIAM J. Comput. 23:1138-1151, 1994.

[15] H.-L. Cheng, T. K. Dey, H. Edelsbrunner, and J. Sullivan. Dynamic skin triangulation. Proc. 12 th Annu. ACM-SIAM Sympos. Discrete Algorithms, 2001. Full version to appear in Discrete Comput. Geom. 〈http://www.cs.duke.edu/ ${ }^{\sim}$ edels/GeoBio/ SkinTri.ps $\rangle$.

[16] S.-W. Cheng, T. K. Dey, H. Edelsbrunner, M. A. Facello, and S.-H. Teng. Sliver exudation. Proc. 15th Annu. Sympos. Comput. Geom., 1-13, 1999.

[17] K. L. Clarkson. Nearest neighbor queries in metric spaces. Discrete Comput. Geom. 22:63-93, 1999.

[18] T. K. Dey, S. Funke, and E. Ramos. Surface reconstruction in almost linear time under locally uniform sampling. Unpublished manuscript, 2001.

[19] R. Dwyer. The expected number of $k$-faces of a Voronoi diagram. Internat. J. Comput. Math. 26(5):13-21, 1993.

[20] R. A. Dwyer. Higher-dimensional Voronoi diagrams in linear expected time. Discrete Comput. Geom. 6:343-367, 1991.

[21] H. Edelsbrunner, X.-Y. Li, G. Miller, A. Stathopolous, D. Talmor, S.-H. Teng, A. Üngör, and N. Walkington. Smoothing and cleaning up slivers. Proc. 32nd Annu. ACM Sympos. Theory Comput., 273-277, 2000.

[22] H. Edelsbrunner and E. P. Mücke. Threedimensional alpha shapes. ACM Trans. Graph. 13(1):43-72, 1994.

[23] C. Gold. Crust and anti-crust: A one-step boundary and skeleton extraction algorithm. Proc. 15th Annu. ACM Sympos. Comput. Geom., 189-196, 1999.

[24] M. Golin and H. Na. On the average complexity of 3d-Voronoi diagrams of random points on convex polytopes. Proc. 12th Canadian Conf. Comput. Geom., 127-135, 2000. 〈http://www.cs.unb.ca/ conf/cccg/eProceedings/44.ps.gz $\rangle$.

[25] B. Guo, J. Menon, and B. Willette. Surface reconstruction using alpha shapes. Comput. Graph. Forum 16(4):177-190, 1997.

[26] H. Hiyoshi and K. Sugihara. Voronoi-based interpolation with higher continuity. Proc. 16th Annu. ACM Sympos. Comput. Geom., 242-250, 2000.

[27] X.-Y. Li and S.-H. Teng. Generating sliver-free three dimensional meshes. Proc. 12th Annu. ACMSIAM Sympos. Discrete Algorithms, 2001.

[28] J. Shewchuk. Tetrahedral mesh generation by Delaunay refinement. Proc. 14th Annu. ACM Sympos. Comput. Geom., 86-95, 1998.
[29] J. Vleugels. On Fatness and Fitness: Realistic Input Models for Geometric Algorithms. Ph.D. Thesis, Dept. Comput. Sci., Univ. Utrecht, 1997. 